BMJ

Open

Gastroenterology

\title{
Acute kidney injury after multiphase imaging for lesions detected on hepatocellular carcinoma surveillance in patients with cirrhosis
}

\author{
Adnan Aman Khan (D , ${ }^{1}$ Yousaf Bashir Hadi, ${ }^{1}$ Jesse Martin Thompson, ${ }^{1}$ \\ Justin Thomas Kupec ${ }^{2}$
}

To cite: Khan AA, Hadi YB, Thompson JM, et al. Acute kidney injury after multiphase imaging for lesions detected on hepatocellular carcinoma surveillance in patients with cirrhosis. BMJ Open Gastro 2020;7:e000394. doi:10.1136/ bmjgast-2020-000394

Received 3 March 2020 Revised 24 March 2020 Accepted 27 March 2020
Check for updates

\section{(c) Author(s) (or their} employer(s)) 2020. Re-use permitted under CC BY-NC. No commercial re-use. See rights and permissions. Published by BMJ.

${ }^{1}$ Department of Medicine, West Virginia University School of Medicine, Morgantown, West Virginia, USA

${ }^{2}$ Section of Gastroenterology \& Hepatology, West Virginia University, Morgantown, West Virginia, USA

Correspondence to Dr Adnan Aman Khan; adnan.aman.khan@gmail.com

\section{ABSTRACT}

Objective The risk difference between multiphase multidetector contrast-enhanced CT and MRI for developing acute kidney injury (AKI) has not been previously evaluated in patients with cirrhosis undergoing hepatocellular carcinoma (HCC) surveillance. We aimed to compare the rate of $\mathrm{AKI}$ after $\mathrm{CT}$ and MRI for evaluation of these lesions.

Design A retrospective chart review of all patients with cirrhosis who underwent either multiphase multidetector liver protocol CT or MRI for lesions detected on HCC screening was conducted at West Virginia University. The rate of $\mathrm{AKI}$ after imaging was compared between the two groups.

Results A total of 416 patients were included. Hepatitis C was the most common aetiology (34.6\%) of cirrhosis. Thirty-six patients had chronic kidney disease at the time of imaging. CT imaging was conducted for 173 (41.5\%) patients, while $58.5 \%$ underwent MRI. Nineteen (4.6\%) patients developed AKI after imaging. The incidence of AKI was $2.89 \%$ for CT and $5.76 \%$ for MRI ( $p$ value $=0.25$ ). Multivariate logistic regression analysis revealed that inpatient status ( $p$ value $=0.015$ ) and Model for End-Stage Liver Disease score ( $p$ value $=0.02$ ) were independently linked to the development of AKI following imaging, while the type of imaging modality was not.

Conclusions There is no difference in the risk of AKI after CT or MRI for evaluation of lesions identified on HCC surveillance. The rates of AKI after these imaging studies are low and are attributable to other aetiologies in most cases. We propose that the choice of imaging should be made based on availability, cost, and other patient-related and facility-related factors.

\section{INTRODUCTION}

Guidelines from major societies including the American Association for the Study of Liver Diseases (AASLD), recommend screening patients with cirrhosis for the development of hepatocellular carcinoma (HCC) with ultrasound imaging. ${ }^{1}$ Lesions detected on ultrasound that warrant further investigation should be evaluated with multiphase multidetector contrast-enhanced computed

\section{Summary box}

What is already known about this subject?

- The American Association for the Study of Liver Diseases recommends routine surveillance of patients with liver cirrhosis for the development of hepatocellular carcinoma (HCC).

- Lesions detected on screening abdominal ultrasound should be further evaluated with multiphase contrast-enhanced CT or MRI.

- The choice of imaging is dictated by multiple factors, including the risk of developing acute kidney injury (AKI); however, there is no current literature comparing the difference in risk between the two modalities.

What are the new findings?

- There is no difference in the risk of developing AKI following CT or MRI in patients with liver cirrhosis undergoing imaging for evaluation of lesions detected on HCC surveillance ultrasound.

How might it impact on clinical practice in the foreseeable future?

- This finding may be of clinical significance when deciding which imaging modality to perform for lesions warranting further investigation on HCC screening. We recommend that the choice of imaging should be made based on availability, cost, other patient and facility-related factors, and not the risk of AKI.

tomography (CECT) or MRI (figure 1). Both imaging modalities offer high sensitivity and specificity for the detection of HCC in patients with cirrhosis found to have liver lesions on ultrasound imaging; however, there is no current consensus regarding which modality is superior. ${ }^{2}$ The choice of imaging modality used to further characterise lesions detected on HCG surveillance is dictated by multiple factors including institutional protocols, provider preference, cost, availability and risk for developing acute kidney injury (AKI) after contrast exposure, among other factors. 


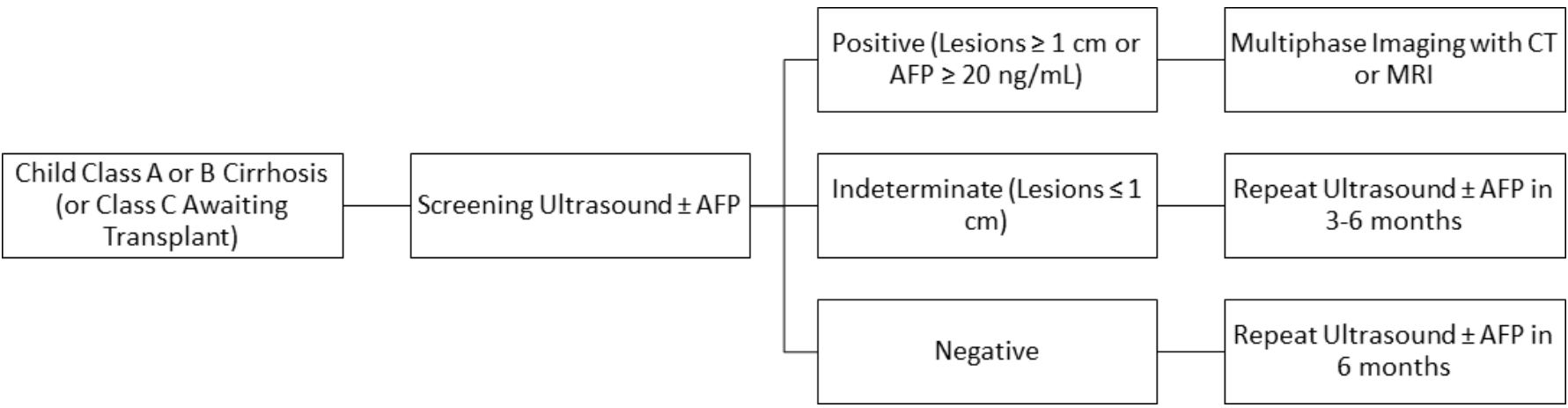

Figure 1 AASLD algorithm for HCC surveillance. AASLD, American Association for the Study of Liver Diseases; AFP, AlphaFetoprotein; HCC, hepatocellular carcinoma.

The development of AKI in patients with cirrhosis is not uncommon and is often a poor prognostic indicator. ${ }^{34}$ Furthermore, there is a significant increase in mortality as the severity of AKI increases. The risk of developing AKI after contrast exposure is an important consideration in these predisposed patients; however, the risk difference between multiphase multidetector CECT and MRI has not been previously explored. We therefore conducted a retrospective cohort study to assess the difference in the risk of developing AKI following these two imaging modalities for further evaluation of lesions detected on HCC surveillance ultrasound.

\section{METHODS}

A retrospective chart review of all patients with cirrhosis who underwent multiphase multidetector CECT or MRI for further evaluation of liver lesions identified on HCC surveillance ultrasound at West Virginia University Medicine (WVUM) was conducted. All patients with cirrhosis who underwent liver protocol MRI or liver protocol CECT imaging between 2010 and 2018 were identified, and their medical charts were reviewed. Patients older than age 18 were included if their imaging was performed for evaluation of lesions identified on HCC surveillance ultrasound and their clinical data were available in our electronic health record system. Medical charts were reviewed to extract demographic and clinical variables including age, gender, comorbid conditions, Model for End-Stage Liver Disease (MELD) scores, type of imaging and development of AKI, among others.

A non-ionic, low-osmolarity iodinated contrast agent 'Iopamidol' is used at our institution for CT imaging. For MRI studies, Gadobutrol is used at our institute, which is a gadolinium-based MRI contrast agent.

AKI was defined and classified using the Kidney Disease Improving Global Outcomes (KDIGO) classification. ${ }^{5}$ KDIGO classification defines AKI as either an increase in serum creatinine by $\geq 0.3 \mathrm{mg} / \mathrm{dL}$ within 48 hours, an increase in serum creatinine $\geq 1.5$ times baseline which is known or presumed to have occurred within the prior 7 days, or decreased urine production to $<0.5 \mathrm{~mL} / \mathrm{kg} / \mathrm{hour}$ for at least 6 hours.
Our study was approved by the Institutional Review Board at WVUM prior to study initiation, and a waiver of informed consent was granted for review of medical records. Data, including baseline demographic characteristics and clinical variables of interest, were extracted by study personnel. Charts were reviewed and clinical notes were examined to identify the development of AKI and its attributed aetiology. Nephrology consultation notes, when available, were also reviewed. Rates of AKI after the two imaging modalities were calculated and compared. Univariate analysis was conducted using t-tests and $\chi^{2}$ tests for continuous and categorical variables, respectively. $\mathrm{P}$ values $<0.05$ were considered significant. A multivariable logistic regression model was then fitted to assess independent associations and control for confounding variables. All analyses were performed using the statistical software R (2019, V.3.6.2). ${ }^{6}$

\section{RESULTS}

A total of 416 patients were included. The sample comprised of a majority of male $(65.4 \%)$ participants with a mean age of 62.6 years and a SD of 10.9. Hepatitis $\mathrm{C}$ was the most common aetiology $(34.6 \%)$ of cirrhosis. Thirty-six $(8.7 \%)$ patients had chronic kidney disease at the time of imaging. No patient with end-stage renal disease met inclusion criteria. Imaging was performed during admission in $126(30.4 \%)$ patients, with 290 $(69.6 \%)$ patients receiving their imaging in the outpatient setting. Mean MELD score of the study population was $13.68( \pm 6.66)$. A majority $(40.89 \%)$ of participants were Child-Pugh Class A, while $32.89 \%$ and $26.22 \%$ of participants were Child-Pugh Class B and C, respectively. Baseline characteristics of the population are summarised in table 1.

CECT imaging was conducted for $173(41.5 \%)$ patients, while the rest $(58.5 \%)$ underwent MRI. Following imaging, 19 (4.6\%) patients developed AKI in the subsequent week. The incidence of AKI following imaging was $2.89 \%$ for the CECT cohort (five patients) and $5.76 \%$ for MRI cohort (14 patients). Mean serum creatinine values prior to and after completion of CECT were $0.92 \mathrm{mg} / \mathrm{dL} \pm 0.42$ and $\quad 1.11 \mathrm{mg} / \mathrm{dL} \pm 0.64, \quad$ respectively; 


\begin{tabular}{|c|c|c|}
\hline Variable & CECT cohort & MRI cohort \\
\hline Age (in years) & $62.26( \pm 11.88)$ & $62.81( \pm 10.19)$ \\
\hline Male gender & $111(64.16 \%)$ & $161(66.26 \%)$ \\
\hline CKD & $19(10.98 \%)$ & $17(7.00 \%)$ \\
\hline \multicolumn{3}{|c|}{ Aetiology of cirrhosis } \\
\hline Alcohol & $59(34.10 \%)$ & $56(23.05 \%)$ \\
\hline $\begin{array}{l}\text { Hepatitis } \mathrm{C} \text { and } \\
\text { alcohol }\end{array}$ & 13 (7.51\%) & $28(11.52 \%)$ \\
\hline Hepatitis B & $2(1.16 \%)$ & $6(2.47 \%)$ \\
\hline Hepatitis C & $35(20.23 \%)$ & 68 (27.98\%) \\
\hline $\mathrm{NASH}$ & $43(24.86 \%)$ & 57 (23.46\%) \\
\hline Unknown & $21(12.14 \%)$ & $28(11.52 \%)$ \\
\hline Inpatient imaging & $45(26.01 \%)$ & 81 (33.33\%) \\
\hline MELD score & $13.92( \pm 6.62)$ & $13.57( \pm 6.70)$ \\
\hline \multirow[t]{2}{*}{$\begin{array}{l}\text { Mean serum } \\
\text { creatinine }\end{array}$} & $\begin{array}{l}\text { Preimaging: } \\
0.92 \mathrm{mg} / \mathrm{dL} \pm 0.42\end{array}$ & $\begin{array}{l}\text { Preimaging: } \\
0.88 \mathrm{mg} / \mathrm{dL} \pm 0.25\end{array}$ \\
\hline & $\begin{array}{l}\text { Postimaging: } \\
1.11 \mathrm{mg} / \mathrm{dL} \pm 0.64\end{array}$ & $\begin{array}{l}\text { Postimaging: } \\
1.07 \mathrm{mg} / \mathrm{dL} \pm 0.55\end{array}$ \\
\hline
\end{tabular}

CECT, contrast-enhanced computed tomography; CKD, chronic kidney disease; MELD, Model for End-Stage Liver Disease; NASH, non-alcoholic steatohepatitis.

values for MRI were $0.88 \mathrm{mg} / \mathrm{dL} \pm 0.25$ and $1.07 \mathrm{mg}$ / $\mathrm{dL} \pm 0.55$, respectively. Univariate analysis using a $\chi^{2}$ test revealed no significant difference in the rate of AKI between the two cohorts ( $p$ value $=0.25)$. On univariate analysis, higher age, inpatient status, MELD score, and Child-Pugh Class at the time of imaging were associated with the development of AKI ( $p$ values of $0.047,<0.001$, 0.002 , and $<0.001$, respectively). Gender ( $p$ value $=0.59$ ), baseline kidney dysfunction ( $p$ value $=0.47$ ), aetiology of cirrhosis $(p$ value $=0.45)$ and type of imaging $(p$ value $=$ 0.25 ) were not significantly associated with development of AKI (table 2).

Multivariate logistic regression analysis was performed incorporating age, gender, inpatient versus outpatient status at time of imaging, baseline kidney dysfunction, type of imaging, and MELD score as covariates. Inpatient status $(p$ value $=0.015)$ and MELD score $(p$ value $=0.02)$ were independently linked to the development of AKI following imaging, while the type of imaging modality was not associated with AKI ( $p$ value $=0.86$ ).

AKI could be attributed to aetiologies other than contrast exposure in 13 out of the 19 patients who developed AKI after imaging. Aetiology of AKI in these cases was prerenal in 10 patients, intrarenal (acute tubular necrosis) in one patient, and hepatorenal syndrome in two patients. No patient was definitively diagnosed with AKI due to contrast exposure. Mean MELD score in the patients who developed AKI was $20.93( \pm 7.42)$, while the mean MELD score was $13.24( \pm 6.37)$ in those who did not develop AKI.

Regarding severity, of the 19 patients who developed AKI after imaging, nine patients developed KDIGO stage 1 AKI, while one patient developed stage 3 AKI. One patient in the CECT cohort who developed AKI required renal replacement therapy (intermittent hemodialysis), with subsequent recovery of renal function. No patient in the MRI cohort required renal replacement therapy.

\section{DISCUSSION}

HCC is among the most diagnosed cancers worldwide and of the few malignancies with an increasing incidence in the USA. Guidelines from major societies recommend screening patients with cirrhosis for HCC; AASLD recommends surveillance of patients with Child-Turcotte-Pugh Class A or B cirrhosis (or Class C awaiting liver transplant) every 6 months with ultrasound imaging alone or in combination with Alpha-Fetoprotein (AFP) testing. ${ }^{7-9}$ Multiphase contrast-enhanced imaging with CT or MRI is recommended in cases of elevated AFP levels $(\geq 20 \mathrm{ng}$ / $\mathrm{mL}$ ), lesions identified on imaging that are $\geq 1 \mathrm{~cm}$ in size, or poor quality ultrasound images. Currently, costeffectiveness and the ability to consistently obtain highquality images have prevented the use of these two modalities as first-line for HCC surveillance. ${ }^{10}$ Among CECT and MRI, guidelines have not recommended any one imaging modality over the other, although multiphase contrast-enhanced imaging is recommended regardless of the type of imaging modality selected. Choice of modality, therefore, is dictated by institutional protocols, provider preference, availability, cost, and

Table 2 Univariate and multivariate analysis for the development of AKI

\begin{tabular}{llll}
\hline Variable & Univariate analysis $\mathbf{P}$ values & Multivariable analysis P values & Odds ratio (95\% Cl) \\
\hline Age & 0.047 & 0.099 & $1.05(0.99$ to 1.12$)$ \\
Male gender & 0.59 & 0.33 & $1.90(0.52$ to 7.00$)$ \\
CKD & 0.47 & 0.74 & $0.74(0.12$ to 4.60$)$ \\
Inpatient imaging & $<0.01$ & 0.015 & $7.54(1.49$ to 38.21$)$ \\
Type of imaging study & 0.25 & 0.82 & $0.86(0.22$ to 3.33$)$ \\
MELD score & 0.002 & 0.021 & $1.10(1.02$ to 1.20$)$ \\
Child-Pugh Class & $<0.001$ & $\begin{array}{l}\text { (not included in multivariable } \\
\text { analyses) }\end{array}$ & (not included in \\
& & multivariable analyses) \\
\hline
\end{tabular}

AKI, acute kidney injury; CKD, chronic kidney disease; MELD, Model for End-Stage Liver Disease. 
patient characteristics, including the risk for developing AKI, among other factors.

Of the two imaging modalities, multiphase multidetector CECT scan is more widely available and costeffective. In addition, it may be performed more promptly and be used in patients with non-MRI compatible metal devices or implants. Recent studies, however, have found MRI to be more sensitive at detecting and characterising smaller lesions, particularly those less than $1-2 \mathrm{~cm}$ in size. ${ }^{11}$ Contrast media used to enhance CT imaging have been previously associated with an increased risk of AKI and are therefore avoided in patients considered to be at high risk.

Previous studies have shown that patients with cirrhosis are at an increased risk for developing AKI and that the development of AKI in these patients is linked to an increase in mortality. ${ }^{34}$ When choosing an imaging modality to further characterise lesions identified on HCC surveillance, the risk of AKI in these patients becomes an important consideration. Conflicting data have been reported in literature regarding the risk of AKI in patients with cirrhosis undergoing CECT imaging. Filomia et al reported an increased incidence of AKI in patients with cirrhosis who underwent inpatient CECT imaging when compared with patients with cirrhosis who did not undergo imaging ( $8.8 \%$ and $3 \%$, respectively). ${ }^{12}$ In the data reported by Campion et al, the incidence of AKI did not differ between hospitalised patients with cirrhosis who underwent CECT imaging, patients with cirrhosis who did not undergo such imaging, and a control group without cirrhosis who underwent CECT imaging. ${ }^{13}$ Only two patients in the study by Filomia $e t a l$, and one patient in Campion et al developed an AKI greater than KDIGO stage $1 .^{12} \mathrm{Ul}$ Abideen et al reported an incidence of $5.1 \%$ in patients with cirrhosis who underwent CECT imaging; however, this study lacked a control group for comparison, and stage of AKI per KDIGO criteria was not discussed. ${ }^{14}$

Our study is the first to report the incidence of AKI in patients with cirrhosis who underwent CECT or MRI imaging for the purpose of HCC surveillance. All patients underwent liver protocol multiphase multidetector CT imaging which requires more contrast exposure as compared with other commonly performed CECT scans. We found an incidence of AKI in the CT cohort that is similar to that reported by Campion et al, and there was no difference in incidence when compared with the MRI group. The rate of AKI after either imaging modality was low, with most patients developing KDIGO stage 1 AKI, similar to previous studies discussed above. Interestingly, most patients who developed AKI in our cohort had confirmed aetiologies other than AKI due to contrast exposure. No patient was definitively diagnosed with AKI due to exposure to contrast. We found a high MELD score to be a risk factor for AKI in patients with cirrhosis after contrasted imaging of the liver. This finding is similar to the observation by previous studies on patients with cirrhosis where higher MELD scores have been reported to be a risk factor for AKI in general, and also after CT imaging. ${ }^{14}$

Our study is limited by its retrospective nature. The amount of contrast administered was not considered, and the study was conducted at a single centre. We were, however, able to draw some pertinent conclusions due to the relatively large number of patients who were included.

In conclusion, we provide evidence that there is no difference in the risk of developing AKI following CECT or MRI in patients with liver cirrhosis undergoing imaging for evaluation of lesions detected on HCC surveillance ultrasound. A higher MELD score was found to be a significant risk factor for the development of AKI. These findings may be of clinical significance when deciding which imaging modality to perform for lesions warranting further investigation on HCC screening. We recommend that the choice of imaging should be made based on availability, cost, and other patient and facilityrelated factors.

Contributors All authors contributed significantly and agree with the content of this manuscript. AAK assisted in conception, project design, data collection, and drafting the manuscript. YBH assisted in conception, project design, data collection, and drafting the manuscript. JMT assisted in statistical analysis and drafting the manuscript. JK assisted in conception, project design, data collection, and drafting the manuscript.

Funding The authors have not declared a specific grant for this research from any funding agency in the public, commercial or not-for-profit sectors.

Competing interests None declared.

Patient consent for publication Not required.

Ethics approval This study was approved by the Institutional Review Board of West Virginia University, protocol ID \#1908661605.

Provenance and peer review Not commissioned; externally peer reviewed.

Data availability statement Data are available upon reasonable request.

Open access This is an open access article distributed in accordance with the Creative Commons Attribution Non Commercial (CC BY-NC 4.0) license, which permits others to distribute, remix, adapt, build upon this work non-commercially, and license their derivative works on different terms, provided the original work is properly cited, appropriate credit is given, any changes made indicated, and the use is non-commercial. See: http://creativecommons.org/licenses/by-nc/4.0/.

\section{ORCID iD}

Adnan Aman Khan http://orcid.org/0000-0001-6724-3988

\section{REFERENCES}

1 Marrero JA, Kulik LM, Sirlin CB, et al. Diagnosis, staging, and management of hepatocellular carcinoma: 2018 practice guidance by the American association for the study of liver diseases. Hepatology 2018;68:723-50.

2 Sherman M, Klein A. AASLD single-topic research conference on hepatocellular carcinoma: conference proceedings. Hepatology 2004;40:1465-73.

3 D'Amico G, Garcia-Tsao G, Pagliaro L. Natural history and prognostic indicators of survival in cirrhosis: a systematic review of 118 studies. J Hepatol 2006;44:217-31.

4 du Cheyron D, Bouchet B, Parienti J-J, et al. The attributable mortality of acute renal failure in critically ill patients with liver cirrhosis. Intensive Care Med 2005;31:1693-9.

5 Khwaja A. KDIGO clinical practice guidelines for acute kidney injury. Nephron Clin Pract 2012;120:c179-84.

6 R Core Team. R: a language and environment for statistical computing. $R$ foundation for statistical computing. Vienna, Austria, 2019. 
7 Heimbach JK, Kulik LM, Finn RS, et al. AASLD guidelines for the treatment of hepatocellular carcinoma. Hepatology 2018;67:358-80.

8 Omata M, Cheng A-L, Kokudo N, et al. Asia-Pacific clinical practice guidelines on the management of hepatocellular carcinoma: a 2017 update. Hepatol Int 2017;11:317-70.

9 Kokudo N, Hasegawa K, Akahane M, et al. Evidence-based clinical practice guidelines for hepatocellular carcinoma: the Japan Society of hepatology 2013 update (3rd JSH-HCC guidelines). Hepatol Res 2015;45.

10 Frenette CT, Isaacson AJ, Bargellini I, et al. A practical guideline for hepatocellular carcinoma screening in patients at risk. Mayo Clin Proc Innov Qual Outcomes 2019;3:302-10.
11 Arif-Tiwari H, Kalb B, Chundru S, et al. MRI of hepatocellular carcinoma: an update of current practices. Diagn Interv Radiol 2014;20:209-21.

12 Filomia R, Maimone S, Caccamo G, et al. Acute kidney injury in cirrhotic patients undergoing contrast-enhanced computed tomography. Medicine 2016;95:e4836.

13 Campion D, Rizzo M, Ponzo P, et al. Risk of contrast-induced acute kidney injury in cirrhotic patients undergoing computed tomography: myth or reality? Dig Liver Dis 2020;52:e64

14 UI Abideen Z, Mahmud SN, Salih M, et al. Contrast-induced acute kidney injury in patients with liver cirrhosis: a retrospective analysis. Cureus 2018;10:e2707. 